\title{
Knockdown of Y-box-binding protein-1 inhibits the malignant progression of HT-29 colorectal adenocarcinoma cells by reversing epithelial-mesenchymal transition
}

\author{
XUE-BING YAN, QING-CHAO ZHU, HONG-QI CHEN, JIA-YUAN PENG, HONG-LEI CHAO, \\ HANG-XIANG DU, ZHI-GANG WANG and ZHI-MING JIN \\ Department of Surgery, Sixth People's Hospital, School of Medicine, \\ Shanghai Jiao Tong University, Shanghai 200233, P.R. China
}

Received October 20, 2013; Accepted May 22, 2014

DOI: $10.3892 / \mathrm{mmr} .2014 .2545$

\begin{abstract}
Y-box binding protein-1 (YB-1) has been identified as an oncoprotein in various malignancies. The aim of this study was to investigate the biological role of YB-1 and its association with epithelial-to-mesenchymal transition (EMT) in colorectal cancer (CRC). The expression of YB-1 and three EMT-related proteins (E-cadherin, N-cadherin and vimentin) was analyzed in $80 \mathrm{CRC}$ and matched normal tissue samples, by immunohistochemistry. The results indicated that the expression of YB-1 was higher in CRC tissue samples than that in matched normal controls and was significantly correlated with tumor differentiation, tumor invasion, lymph node metastasis and distant metastases. Furthermore, analysis showed that YB-1 expression was negatively correlated with E-cadherin and positively correlated with $\mathrm{N}$-cadherin and vimentin expression. In vitro assays showed that knockdown of YB-1 inhibited the proliferation, apoptosis resistance, invasion and migration of the HT-29 CRC cell line. Of note, following knockdown of YB-1, E-cadherin expression was elevated whereas $\mathrm{N}$-cadherin and vimentin expression was reduced. Taken together, these results suggest that YB-1 promotes the malignant progression of $\mathrm{CRC}$ in part through the induction of EMT, and YB-1 may therefore be a potential novel target for $\mathrm{CRC}$ treatment.
\end{abstract}

\section{Introduction}

Despite ongoing advances in diagnosis and treatment, colorectal cancer (CRC) remains a common fatal cancer affecting both genders that contributes to an estimated 600,000 mortalities

Correspondence to: Dr Zhi-Ming Jin, Department of Surgery, Sixth People's Hospital, School of Medicine, Shanghai Jiao Tong University, 600 Yishan Road, Shanghai 200233, P.R. China

E-mail: jzmgyp@aliyun.com

Key words: YB-1, colorectal cancer, epithelial-mesenchymal transition, invasion, metastasis worldwide annually (1). Among the various factors affecting the chance of survival in patients with $\mathrm{CRC}$, the formation of distant metastases is the most dominant. Various proteins have been demonstrated to be dysregulated and function in the metastasis of CRC; however, the molecular mechanisms and signaling pathways involved require further study.

Y-box binding protein-1 (YB-1) is a member of the cold-shock protein superfamily. The protein is encoded by the YBX1 gene and contains a highly conserved nucleic-acid-binding motif that binds to both DNA and RNA. YB-1 functions within the cytoplasm and nucleus with roles in transcriptional regulation, DNA repair and replication and pre-mRNA splicing. Recent studies have regarded YB-1 as an oncoprotein due to its involvement in uncontrolled proliferation (2), apoptosis resistance (3), sustained angiogenesis (4), invasion and metastasis (5). Furthermore, YB-1 has been associated with chemotherapeutic resistance (6) and high YB-1 expression is closely linked to the poor clinical outcome in various types of cancer, including gastric (7), ovarian (8) and prostate (9). Other studies have suggested that the carcinogenic effect of YB-1 may be associated with epithelial-to-mesenchymal transition (EMT) $(10,11)$.

EMT is a well-known regulatory program involved in embryonic development, wound healing and tumor development (12). Through this program, epithelial cells lose apical-basal polarity and tight junctions, thus acquiring a mesenchymal phenotype that is more amenable to cell migration and invasion. Molecularly, EMT is characterized by a downregulation of epithelial markers and an upregulation of mesenchymal markers. E-cadherin is a well-known epithelial marker that hinders the movement of epithelial cells by mediating adherens junctions (13). The loss of E-cadherin has been regarded to have an unfavorable effect on the overall survival of patients with CRC, as described by He et al (14). Furthermore, emerging studies have suggested that mesenchymal markers, such as vimentin and $\mathrm{N}$-cadherin, promote the metastasis of CRC and act as novel prognostic factors $(15,16)$.

A close association between YB-1 and EMT was first identified by Evdokimova et al (17), who showed that YB-1 could promote EMT in premalignant breast epithelial cells 
in vivo and in vitro. Twist-related protein 1 (Twist1) is another well-established EMT transcription factor, and it was shown by Shiota et al (18) that a mutual regulation of Twist1 and YB-1 could promote the malignant progression of bladder cancer, again suggesting an involvement of YB-1 in EMT. However, neither the biological role of YB-1 nor its association with EMT has been sufficiently studied in CRC. In this study, the association between the expression of YB-1 and three EMT markers (E-cadherin, $\mathrm{N}$-cadherin and vimentin) in human CRC tissues was analyzed. Small interfering RNA (siRNA) was employed to explore the specific roles of YB-1 in a CRC cell line, HT-29, well-characterized for use in EMT-associated assays in vitro and in vivo $(19,20)$. The aim of the study was to investigate the hypotheses that i) YB-1 may be associated with a dysregulated EMT phenotype in CRC and ii) YB-1 may promote the growth, invasion and migration of CRC cells, in part through the induction of EMT.

\section{Materials and methods}

Tissue samples and patient data. A total of 80 primary CRC tumors and corresponding normal tissues were collected from patients with CRC undergoing surgery at the Department of Surgery, The Sixth People's Hospital affiliated to Shanghai Jiao Tong University (Shanghai, China), between 2011 and 2012. None of the patients had received preoperative chemotherapy or radiotherapy. The study was approved by the Ethics Committee of The Sixth People's Hospital affiliated to Shanghai Jiao Tong University. Written informed consent was obtained from patients for use of their tissue specimens in the study. The basic clinical characteristics of the patients are shown in Table I.

Immunohistochemistry. Paraffin-embedded tissue specimens were cut at $4-\mu \mathrm{m}$-thickness. Following deparaffinization and dehydration, the sections were placed in citrate buffer solution and heated using a microwave oven for antigen retrieval. Following this, $0.3 \%$ hydrogen peroxidase and methanol were used to block endogenous peroxidase activity for $25 \mathrm{~min}$ and the sections were incubated with primary antibodies against YB-1 (1:200, Epitomics, Burlingame, CA, USA), E-cadherin (1:150, Epitomics), N-cadherin (1:150, Epitomics) and vimentin (1:200, Bioworld, St. Louis Park, MN, USA) at $4^{\circ} \mathrm{C}$ overnight. EnVision $^{\mathrm{TM}}$ reagents (Dako, Glostrup, Denmark) were used to detect antibody binding. The sections were then counterstained with hematoxylin and observed by light microscopy. The immunostaining evaluation was performed by two independent researchers, who were blinded to the clinicopathological parameters of the patients. The evaluation principle used was that described in the study by Wu et al (21).

Cell culture. The HT-29 human colon adenocarcinoma cell line was obtained from the Type Culture Collection of the Chinese Academy of Sciences (Shanghai, China). The cells were maintained in McCoy's 5A culture medium (Sigma-Aldrich, St. Louis, MO, USA) supplemented with $10 \%$ fetal bovine serum (FBS; Gibco-BRL, Grand Island, NY, USA) and $1 \%$ penicillin/streptomycin (Gibco-BRL) at $37^{\circ} \mathrm{C}$ in a humidified atmosphere containing $95 \%$ air and $5 \% \mathrm{CO}_{2}$. The medium was changed every two days.
YB-1 RNA interference. Commercially generated siRNA (GenePharma, Shanghai, China) was used to decrease YB-1 expression in HT-29 cells. A negative random siRNA that did not target any human gene was used as a negative control. The sequences for siRNA and control siRNA are shown in Table II. The cells were divided into three groups: Control (cells with transfection reagent only), siControl (cells treated with negative control siRNA and transfection reagent) and siYB-1 (cells treated with siRNA and transfection reagent).

Transfection was performed using Lipofectamine ${ }^{\mathrm{TM}} 2000$ transfection reagent (Invitrogen Life Technologies, Carlsbad, CA, USA). Briefly, 3,000 cells per well were seeded in a 96-well plate $24 \mathrm{~h}$ before transfection. The siRNAs were then diluted in serum-free medium and mixed with the transfection reagent that was pre-diluted in an equal volume of serum-free medium. The mixture was then added to each well. Following incubation at $37^{\circ} \mathrm{C}$ for $6 \mathrm{~h}$, the cell medium was changed to complete medium. For the following assays, the reverse transcription polymerase chain reaction (RT-PCR) was performed $24 \mathrm{~h}$ after transfection and western blotting, MTT, apoptosis and Transwell ${ }^{\mathrm{TM}}$ assays were performed $48 \mathrm{~h}$ after transfection.

Quantitative (q)PCR. Total RNA was isolated from cells using TRIzol ${ }^{\circledR}$ reagent (Invitrogen Life Technologies) according to the manufacturer's instructions. The obtained RNA was used to synthesize cDNA by Superscript III Reverse Transcriptase (Promega Corp., Madison, WI, USA). qPCR reaction mixes were prepared using SYBR Green (Takara, Shiga, Japan) and run on the StepOne ${ }^{\mathrm{TM}}$ Plus Real-Time PCR system (Applied Biosystems, Foster City CA, USA) with the following conditions: $95^{\circ} \mathrm{C}$ for $5 \mathrm{~min}, 95^{\circ} \mathrm{C}$ for $5 \mathrm{sec}$ and $60^{\circ} \mathrm{C}$ for $30 \mathrm{sec}$, for 40 cycles. The relative mRNA expression value was calculated by the $2^{-\Delta \Delta \mathrm{T}}$ method, and $\beta$-actin expression was taken as an internal control. The sequences of the primers used are shown in Table III.

Western blot analysis. Total protein was extracted from the cells using lysis buffer $(50 \mathrm{mmol} / \mathrm{L}$ Tris- $\mathrm{HCl}, \mathrm{pH} 8.0$; $150 \mathrm{mmol} / \mathrm{L} \mathrm{NaCl} ; 1 \%$ TritonX-100; $100 \mu \mathrm{g} / \mathrm{ml} \mathrm{PMSF})$. The lysates containing $20 \mu \mathrm{g}$ protein were separated by $12 \%$ SDS-PAGE and transferred onto polyvinylidene difluoride membranes. The membranes were blocked for $1 \mathrm{~h}$ at room temperature in blocking solution [5 g skimmed milk powder and $100 \mathrm{ml}$ Tris-buffered saline with $0.1 \%$ Tween 20 (TBST)]. The membranes were then incubated overnight in primary antibody [anti-YB-1 (1:2,000), -E-cadherin $(1: 2,000)$, -N-cadherin $(1: 5,000)$ and -vimentin $(1: 5,000)]$ at $4^{\circ} \mathrm{C}$. Following three washes with TBST, the membranes were incubated with horseradish peroxidase-conjugated secondary antibody for $2 \mathrm{~h}$ at room temperature. Chemiluminescence reagent (Santa Cruz Biotechnology, Inc., Dallas, TX, USA) was used to detect the protein expression according to the manufacturer's instructions.

MTT assay. Cell proliferation was determined by an MTT assay. Cells (3,000 per well) were incubated with $150 \mu 1$ complete media containing $0.5 \mathrm{mg} / \mathrm{ml}$ MTT for 3-4 h until purple precipitate was visible. Dimethylsulfoxide was used to dissolve the precipitate and the plate was agitated gently for 
Table I. Association between YB-1 expression and clinicopathological parameters.

\begin{tabular}{|c|c|c|c|c|}
\hline Variables & Number & Low YB-1 expression (n) & High YB-1 expression (n) & P-value \\
\hline Total & 80 & 27 & 53 & \\
\hline \multicolumn{5}{|l|}{ Gender } \\
\hline Male & 47 & 14 & 33 & \multirow[t]{2}{*}{0.371} \\
\hline Female & 33 & 13 & 20 & \\
\hline \multicolumn{5}{|l|}{ Age in years } \\
\hline$\leq 60$ & 31 & 12 & 19 & \multirow[t]{2}{*}{0.456} \\
\hline$>60$ & 49 & 15 & 34 & \\
\hline \multicolumn{5}{|l|}{ Tumor location } \\
\hline Colon & 50 & 18 & 32 & \multirow[t]{2}{*}{0.583} \\
\hline Rectum & 30 & 9 & 21 & \\
\hline \multicolumn{5}{|l|}{ Tumor differentiation } \\
\hline Well & 28 & 15 & 13 & \multirow[t]{2}{*}{0.006} \\
\hline Moderate and poorly & 52 & 12 & 40 & \\
\hline \multicolumn{5}{|l|}{ Tumor invasion } \\
\hline pT1-T2 & 35 & 17 & 18 & \multirow[t]{2}{*}{0.013} \\
\hline pT3-T4 & 45 & 10 & 35 & \\
\hline \multicolumn{5}{|l|}{ Lymph node metastasis } \\
\hline Present & 44 & 8 & 36 & \multirow[t]{2}{*}{0.001} \\
\hline Absent & 36 & 19 & 17 & \\
\hline \multicolumn{5}{|l|}{ Distant metastasis } \\
\hline Present & 42 & 9 & 33 & \multirow[t]{2}{*}{0.014} \\
\hline Absent & 38 & 18 & 20 & \\
\hline
\end{tabular}

YB-1, Y-box binding protein-1.

Table II. siRNA sequences.

\begin{tabular}{lll}
\hline Gene & Orientation & Sequence $\left(5^{\prime}-3^{\prime}\right)$ \\
\hline YB-1-homo-375 (siRNA1) & Forward & GGAACGGAUAUGGUUUCAUTT \\
YB-1-homo-529 (siRNA2) & Reverse & AUGAAACCAUAUCCGUUCCTT \\
YB-1-homo-628 (siRNA3) & Forward & GGAGGCAGCAAAUGUUACATT \\
YB-1-homo-940 (siRNA4) & Reverse & UGUAACAUUUGCUGCCUCCTT \\
& Forward & GGUCCUCCACGCAAUUACCAGCAAA \\
Control siRNA & Reverse & UUUGCUGGUAAUUGCCUGGAGGACC \\
& Forward & GGACGGCAAUGAAGAAGAUTT \\
\hline
\end{tabular}

YB-1, Y-box binding protein-1; siRNA, small interfering RNA.

$10 \mathrm{~min}$. The absorbance of each well was then measured using a microplate reader (Molecular Devices, Sunnyvale, CA, USA) at a wavelength of $490 \mathrm{~nm}$.

Apoptosis assay. Flow cytometry using propidium iodide (PI) staining was employed to determine the effect of YB-1 on cell apoptosis. The cells $\left(1 \times 10^{6}\right)$ were washed twice with phosphate-buffered saline and resuspended in $500 \mu \mathrm{l}$ binding buffer containing $5 \mu \mathrm{l}$ Annexin V-fluorescein isothiocyanate (FITC). A total of $5 \mu \mathrm{l}$ PI was then added to the cells and incubated at room temperature for $20 \mathrm{~min}$. The apoptotic cells were subsequently counted by flow cytometry. The Annexin V-FITC Cell Apoptosis Analysis kit was purchased from KeyGen Biotech (Nanjing, China). 
Table III. Primer sequences for the quantitative polymerase chain reaction.

\begin{tabular}{|c|c|c|c|}
\hline Gene & Orientation & Sequence $\left(5^{\prime}-3^{\prime}\right)$ & Length (bp) \\
\hline YB-1 & $\begin{array}{l}\text { Forward } \\
\text { Reverse }\end{array}$ & $\begin{array}{l}\text { TACCTTCGCAGTGTAGGAGAT } \\
\text { ACCACCAGG ACCTGTAACAT }\end{array}$ & 96 \\
\hline E-cadherin & $\begin{array}{l}\text { Forward } \\
\text { Reverse }\end{array}$ & $\begin{array}{l}\text { AAG ACA AAG AAGGCA AGGT } \\
\text { AGAGAGTGTATGTGGCAATG }\end{array}$ & 147 \\
\hline $\mathrm{N}$-cadherin & $\begin{array}{l}\text { Forward } \\
\text { Reverse }\end{array}$ & $\begin{array}{l}\text { CAAGATGGGTCA ATGGAA ATAG } \\
\text { CTCAGGAATACGAGCCTTCAC }\end{array}$ & 174 \\
\hline Vimentin & $\begin{array}{l}\text { Forward } \\
\text { Reverse }\end{array}$ & $\begin{array}{l}\text { CTGTAAGTTGGTAGCACTGAG } \\
\text { TTAGGGGAA ACCGTTAGAC }\end{array}$ & 84 \\
\hline$\beta$-actin & $\begin{array}{l}\text { Forward } \\
\text { Reverse }\end{array}$ & $\begin{array}{l}\text { AAGGTGACAGCAGTCGGTT } \\
\text { TGTGTGGACTTGGGAGAGG }\end{array}$ & 195 \\
\hline
\end{tabular}

YB-1, Y-box binding protein-1; bp, base pairs.

Table IV. Correlation between YB-1 and EMT markers.

\begin{tabular}{|c|c|c|c|c|c|}
\hline \multirow[b]{2}{*}{ EMT markers } & \multirow[b]{2}{*}{$\mathrm{N}$} & \multicolumn{2}{|c|}{ YB-1 expression } & \multirow[b]{2}{*}{ r } & \multirow[b]{2}{*}{ P-value } \\
\hline & & Low (n) & $\operatorname{High}(\mathrm{n})$ & & \\
\hline \multicolumn{6}{|l|}{ E-cadherin } \\
\hline Low & 58 & 14 & 44 & -0.330 & 0.003 \\
\hline High & 22 & 13 & 9 & & \\
\hline \multicolumn{6}{|l|}{$\mathrm{N}$-cadherin } \\
\hline Low & 32 & 17 & 15 & 0.335 & 0.002 \\
\hline High & 48 & 10 & 38 & & \\
\hline \multicolumn{6}{|l|}{ Vimentin } \\
\hline Low & 24 & 16 & 8 & 0.456 & $<0.001$ \\
\hline High & 56 & 11 & 45 & & \\
\hline
\end{tabular}

EMT, epithelial-to-mesenchymal transition; YB-1, Y-box binding protein-1.

Transwell assay. Transwell assays were performed to assess cell migration and invasion using Transwell chambers (BD Biosciences, San Jose, CA, USA). For the Transwell assay without Matrigel $^{\mathrm{TM}}$, cells resuspended in $150 \mu \mathrm{l}$ serum-free medium were added into the upper chamber and $600 \mu 1$ McCoy's 5A culture medium with $10 \%$ FBS was added into the lower chamber. Following incubation for $24 \mathrm{~h}$ at $37^{\circ} \mathrm{C}$ with $5 \% \mathrm{CO}_{2}$ humidified conditions, non-migrating cells in the upper chamber were scraped using a cotton swab. The migrating cells were fixed with $4 \%$ polyoxymethylene for $15 \mathrm{~min}$ and stained with crystal violet for $10 \mathrm{~min}$. Finally, the stained cells from six random fields were counted, and images were captured under a light microscope.

For the Transwell assay with Matrigel, $50 \mu 1$ pre-diluted Matrigel was added into the chambers and incubated at $37^{\circ} \mathrm{C}$ for $2 \mathrm{~h}$. The assay was then performed using the method described for the Transwell assay without Matrigel.

Statistical analysis. All assays were repeated three times independently. The results are presented as the mean \pm standard deviation. SPSS 16.0 statistical software (SPSS, Inc., Chicago, IL, USA) was used to perform all the statistical analyses. The data from the cell assays were analyzed by Student's t test. The correlation between YB-1 expression and clinicopathological parameters was determined by $\chi^{2}$ test, while the correlation between YB-1 and EMT-related markers was determined by nonparametric Spearman's rank correlation coefficient (r). $\mathrm{P}<0.05$ was considered to indicate a statistically significant difference.

\section{Results}

Expression of YB-1 and EMT markers is correlated in CRC tissues. By immunohistochemical analysis, strong YB-1 expression was observed in 66.3\% (53/80) of tumors, compared with $22.5 \%$ (18/80) of the matched normal tissues (Table IV). To determine whether YB-1 was correlated with an EMT phenotype, the expression of three EMT markers (E-cadherin, N-cadherin and vimentin) was investigated (Fig. 1). The expression of E-cadherin, an epithelial marker, was weak in $72.5 \%(58 / 80)$ of 
A YB-1
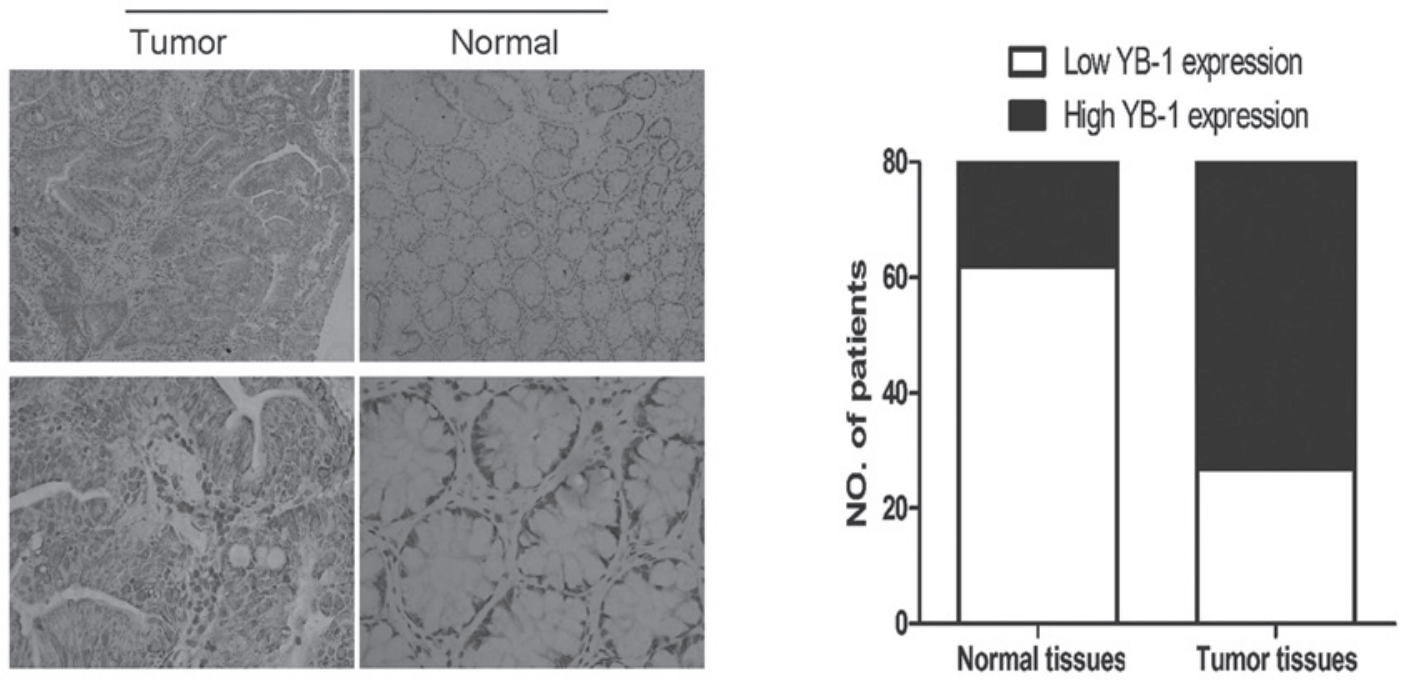

B

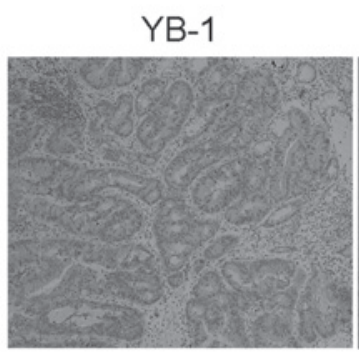

E-cadherin
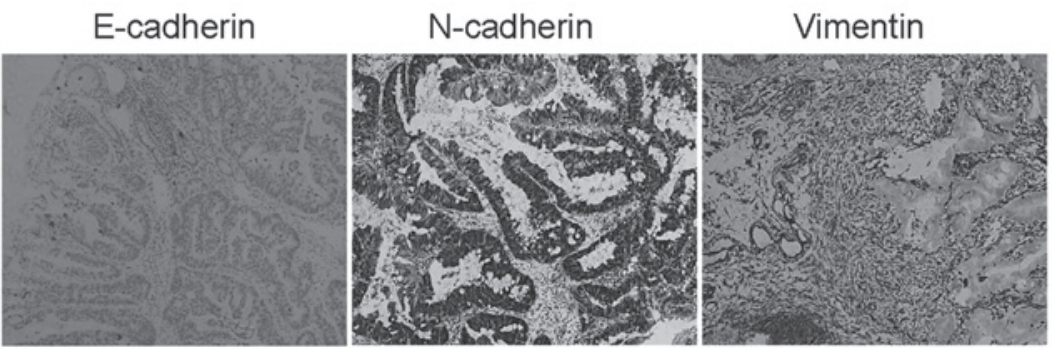

Figure 1. Expression of YB-1 and epithelial-to-mesenchymal transition-related markers in CRC. (A) Expression of YB-1 was higher in tumor tissues than that in matched normal tissues (upper row original magnification, x100; lower row original magnification, $\mathrm{x} 400$ ). (B) A representative patient was shown to have high expression of YB-1, N-cadherin and vimentin, but low expression of E-cadherin in CRC tissues (original magnification, x100). YB-1, Y-box binding protein-1; CRC, colorectal cancer.

tumors, whereas strong expression of two mesenchymal markers ( $\mathrm{N}$-cadherin and vimentin) was detected in $60.0 \%$ (48/80) and $70.0 \%$ (56/80) of the tumors, respectively (Table IV). Correlation analysis showed that YB-1 expression was negatively correlated with E-cadherin expression $(r=-0.330, P=0.003)$, but positively correlated with the expression of $\mathrm{N}$-cadherin $(\mathrm{r}=0.335, \mathrm{P}=0.002)$ and vimentin $(\mathrm{r}=0.456, \mathrm{P}<0.001)$.

YB-1 expression is associated with clinicopathological parameters. The association between YB-1 and clinicopathological parameters of patients with CRC is shown in Table I. High YB-1 expression was shown to have a statistically significant association with tumor differentiation $(\mathrm{P}=0.006)$, tumor invasion $(\mathrm{P}=0.013)$, lymph node metastasis $(\mathrm{P}=0.001)$ and distant metastasis $(\mathrm{P}=0.014)$. However, no correlation was observed between strong YB-1 expression and other parameters, including age $(\mathrm{P}=0.456)$, gender $(\mathrm{P}=0.371)$ and tumor location $(\mathrm{P}=0.583)$.

Knockdown of YB-1 inhibits proliferation and enhances apoptosis in the HT-29 cell line. To explore the specific biological function of YB-1 in CRC, siRNA was used to knock down YB-1 in HT-29 cells. RT-PCR was employed to determine the transfection efficiency of the siRNAs and showed that the YB-1 mRNA expression was reduced by varying degrees using different siRNA sequences (Fig. 2A). Compared with the siControl group, siRNA1 reduced YB-1 mRNA expression by $27.6 \%$, siRNA 2 by $67.1 \%$ and siRNA3 by $49.8 \%$. No statistically significant difference was observed between the siRNA control and siRNA4 ( $P>0.05)$. As a result, siRNA2 was selected for the subsequent in vitro assays.

It is widely accepted that uncontrolled proliferation and resistance to apoptosis are basic features of malignancy. In this study, cell proliferation was measured using an MTT assay. The proliferation of the cells treated with siRNA to downregulate YB-1 expression (siYB-1 group) was significantly inhibited as compared with the control and siControl groups $(\mathrm{P}<0.01$, Fig. 2B). The number of apoptotic cells was next calculated by flow cytometry. It was observed that downregulation of YB-1 by siRNA resulted in an increased proportion of apoptotic cells as compared with the other two groups (apoptotic rate, $22.3 \pm 1.3$ vs. $10.6 \pm 0.7$ and $11.3 \pm 0.9 \%$; siYB-1 group versus the control and siControl groups, respectively; $\mathrm{P}<0.01$; Fig. 2C). These results suggest that YB-1 may promote the growth of HT-29 cells.

Knockdown of YB-1 inhibits the migration and invasion of HT-29 cells. Invasion and metastasis are critical factors associated with the high fatality rate in patients with malignant tumors. Through the Transwell migration assay, it was calculated that the number of migratory cells was significantly decreased in the siYB-1 group, as compared with that in the other two groups $(24.4 \pm 2.6$ vs. $42.1 \pm 6.4$ and $38.9 \pm 4.6 /$ field; siYB-1 group versus the control and siControl groups, respectively; $\mathrm{P}<0.05$; Fig. $3 \mathrm{~A}$ ). Using a Transwell 
A

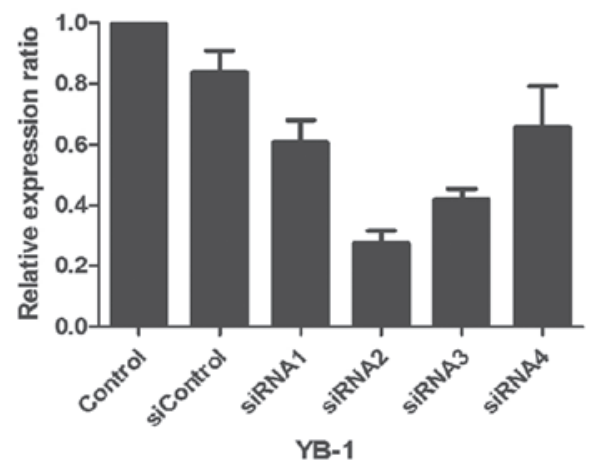

C

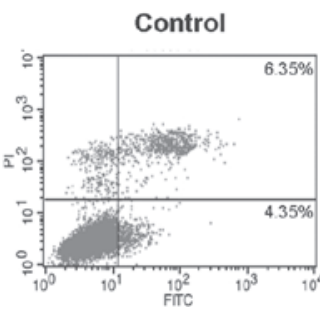

siControl

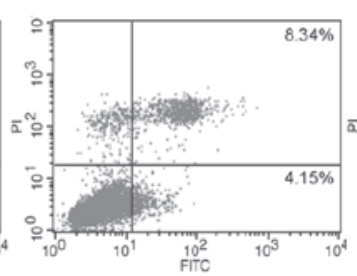

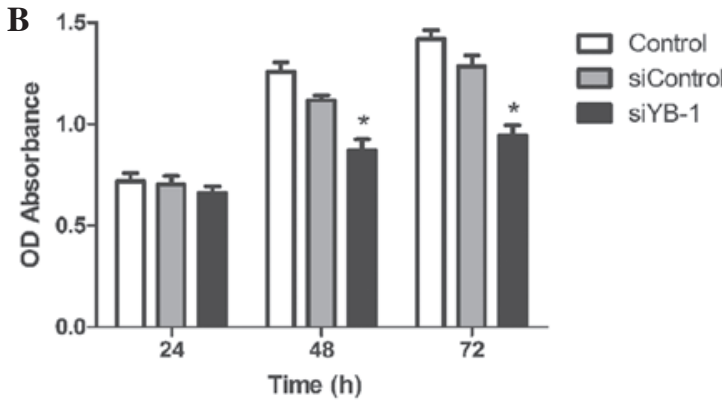

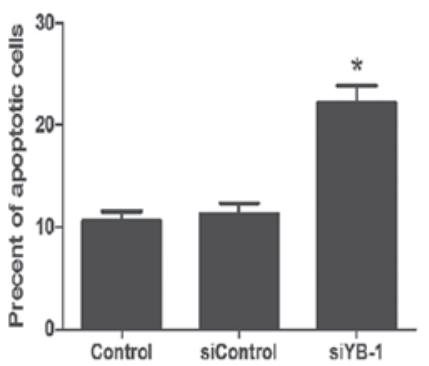

Figure 2. Proliferation and apoptotic ability of HT-29 cells subsequent to knockdown of YB-1. (A) The mRNA expression of YB-1 was reduced by varying degrees by different siRNAs (siRNA1-4). (B) MTT assay. Cell proliferation was inhibited following knockdown of YB-1, compared with the control and siControl group $\left({ }^{*} \mathrm{P}<0.01\right)$. (C) Apoptosis assay. The left three panels are representative results of the flow cytometry. The bar chart shows that knocking down YB-1 increased the apoptosis rate of HT-29 cells, as compared with the control and the siControl group ( $\left.{ }^{*} \mathrm{P}<0.01\right)$. siRNA, small interfering RNA; siControl, siRNA control; YB-1, Y-box binding protein 1; siYB-1, siRNA targeting YB-1; OD, optical density; FITC, fluorescein isothiocyanate; PI, propidium iodide.

A

\section{Control}

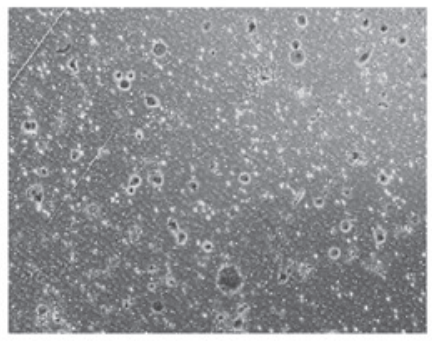

B

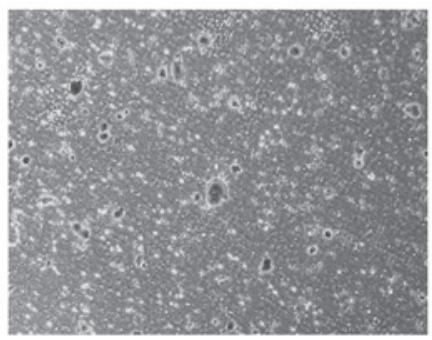

siControl

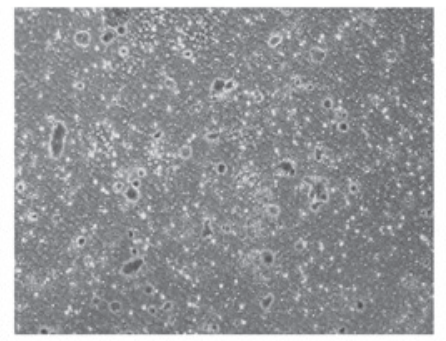

siControl

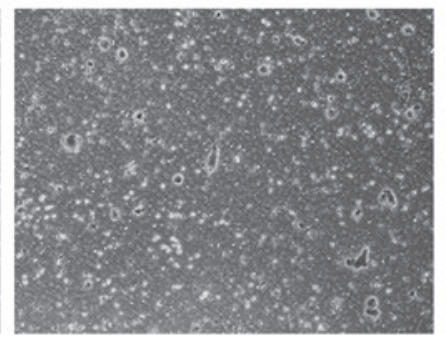

siYB-1

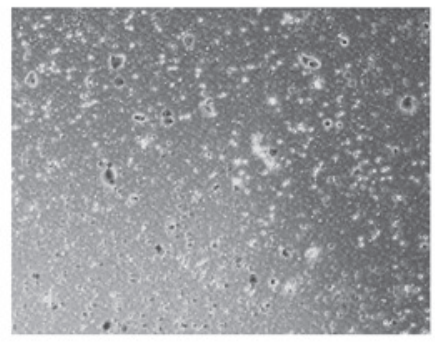

siYB-1

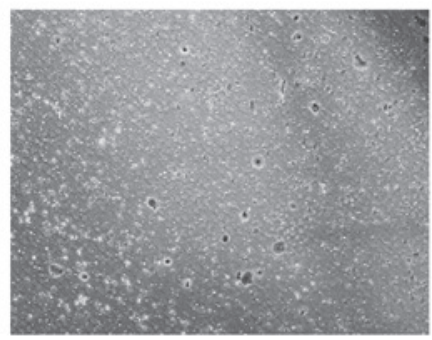

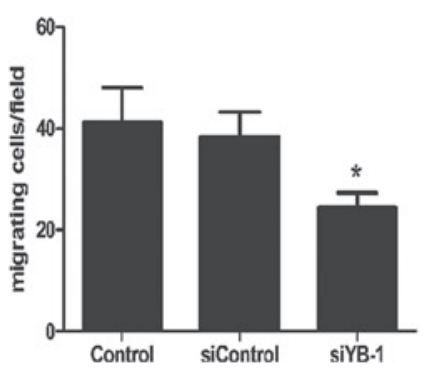

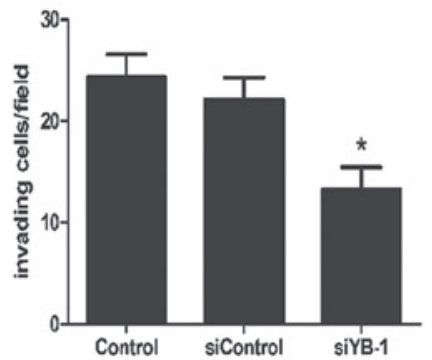

Figure 3. Migratory and invasive ability of HT-29 cells subsequent to knockdown of YB-1. (A) Transwell migration assay. The number of migratory cells (stained with crystal violet) was decreased following knockdown of YB-1, compared with the control and siControl groups ("P<0.01). (B) Transwell invasion assay. The number of invasive cells (stained with crystal violet) across Matrigel was decreased following knockdown of YB-1, as compared with the control and siControl groups ("P<0.01). YB-1, Y-box binding protein-1; siRNA, small interfering RNA; siControl, siRNA control; siYB-1, siRNA targeting YB-1.

invasion assay method, it was also found that the cells in the siYB-1 group exhibited a significant decrease in the number of cells invading across the Matrigel (13.3 \pm 1.9 vs. $24.5 \pm 2.0$ and $22.5 \pm 1.8 /$ field; siYB-1 group versus the control and siControl groups, respectively; $\mathrm{P}<0.01$; Fig. 3B). Collectively, this suggested that YB-1 may contribute to the invasion and migration of HT-29 cells.
Knockdown of $Y B-1$ reverses the EMT phenotype. Since EMT is defined as a key molecular event promoting tumor progression, it was investigated whether YB-1 could promote the EMT program in HT-29 cells. Through qPCR, it was observed that the mRNA levels of E-cadherin were increased by $53.9 \%$, compared with those in the siControl group. Conversely, the mRNA levels of $\mathrm{N}$-cadherin and vimentin 

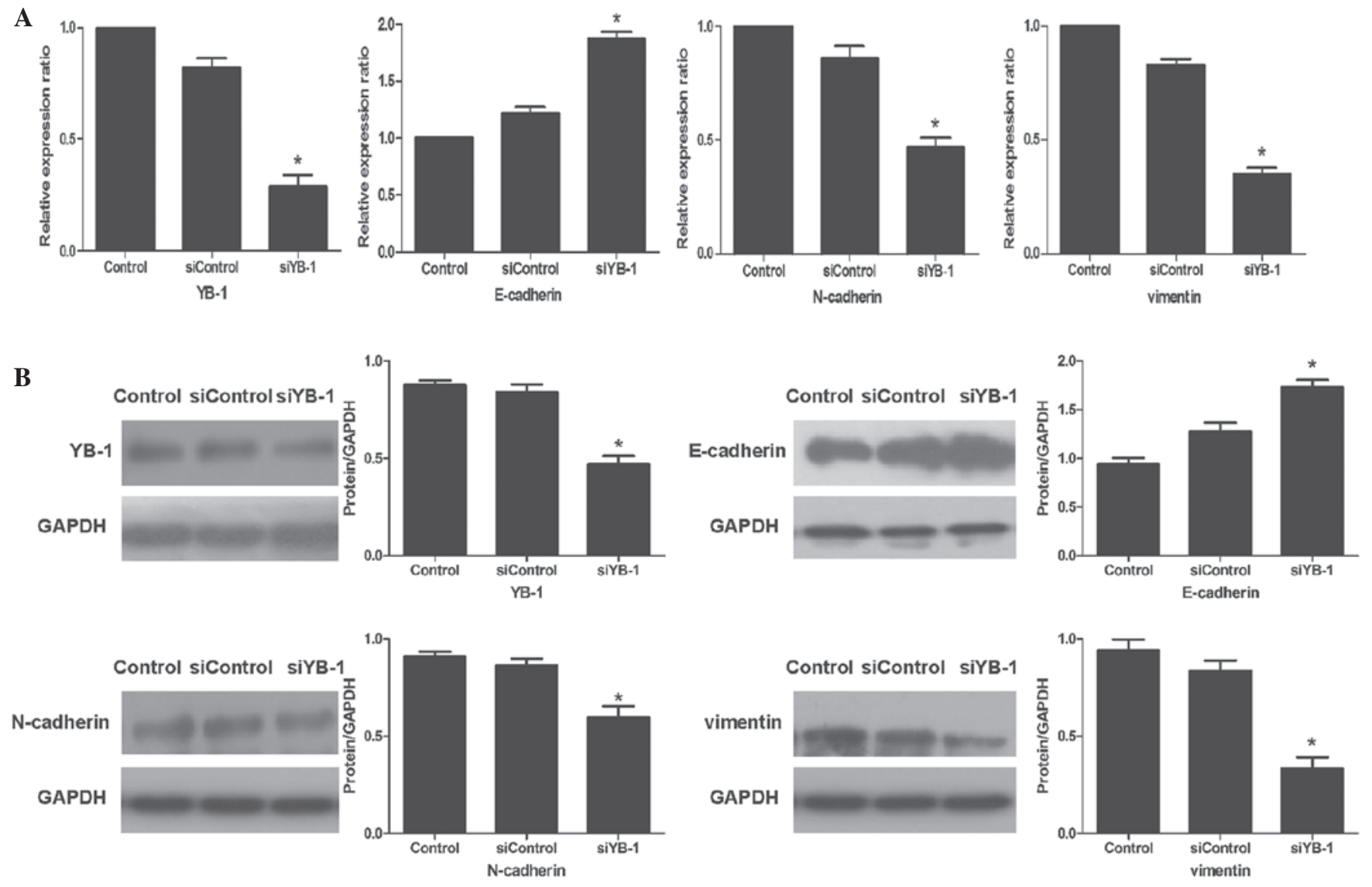

Figure 4. Downregulating YB-1 causes changes in the expression of EMT-related markers. (A) Quantitative polymerase chain reaction. Following YB-1 inhibition, the expression of E-cadherin was increased, whereas the expression of N-cadherin and vimentin was decreased in HT-29 cells, as compared with the control and siControl groups ( $\mathrm{P}<0.01)$. (B) Western blotting results matched those of the quantitative polymerase chain reaction assay ("P<0.01). $\mathrm{YB}-1$, Y-box binding protein-1; siRNA, small interfering RNA; siControl, siRNA control; siYB-1, siRNA targeting YB-1.

were decreased by 45.4 and $57.9 \%$, respectively (Fig. 4A). These results were confirmed by western blotting (Fig. 4B). Based on these findings, it can be speculated that YB-1 can promote the invasion and metastasis of HT-29 cells by regulating the EMT program.

\section{Discussion}

Research over the past decades has elucidated the roles of oncogenes and epigenetic changes in the malignant progression of CRC. Recent studies have investigated the underlying molecular mechanisms in CRC, and particularly those associated with the EMT, which regulate cell survival (22), drug resistance (23), invasion and metastasis (24). A further understanding of the initiating factors that regulate EMT mechanisms is likely to be beneficial in the development of targeted therapy for CRC.

YB-1 has been described as a versatile oncoprotein and has been shown to facilitate numerous hallmarks of cancer (25). However, research investigating the specific biological role of YB-1 in CRC is still in its infancy. Tsofack et al (26) found that overexpressing YB-1 in two CRC cell lines (SW480 and HT-29) could induce resistance to oxaliplatin; this process could be blocked by knockdown of non-POU domain containing octamer-binding protein and RALY heterogeneous nuclear ribonucleoprotein. Jürchott et al (27) demonstrated that YB-1 could activate the mitogen-activated protein kinase kinase/extracellular signal-regulated kinase signaling pathway by regulating cyclin B1 in HCT116 colon cancer cells and that nuclear YB-1 expression was associated with pulmonary metastasis. However, neither of these studies reported the role of YB-1 in EMT. Considering the efforts of the study by Evdokimova et al (17) in breast cancer, this study aimed to investigate whether YB-1 is also crucial for proliferation, apoptosis resistance, invasion and migration in CRC and whether these malignant characteristics are caused by the regulation of YB-1 in the EMT program.

Firstly, the expression of YB-1 in human CRC tissues and matched normal tissues was analyzed. It was found that YB-1 was overexpressed in tumor tissues and was significantly correlated with tumor differentiation and invasion, lymph node metastasis and distant metastasis. No significant correlation was observed between YB-1 expression and other clinicopathological factors, including gender, age and tumor location. To investigate whether YB-1 expression was associated with the characteristics of EMT, correlation analysis between YB-1 and three widely acknowledged EMT markers was performed. It was shown that YB-1 expression was negatively correlated with E-cadherin expression, but positively correlated with the expression of $\mathrm{N}$-cadherin and vimentin. This observation suggested a potential involvement of YB-1 in the EMT program. 
To further investigate the role of YB-1 in the malignant potential of CRC, YB-1 was silenced using siRNA in the HT-29 colon cancer cell line. Silencing YB-1 induced changes in cancer cells resembling those of reversing the EMT program. Knockdown of YB-1 i) inhibited cell proliferation and enhanced apoptosis; ii) inhibited cell invasion and migration; iii) upregulated E-cadherin and downregulated $\mathrm{N}$-cadherin and vimentin. Taken together, these data indicate that YB-1 may drive malignant progression partly by regulating EMT in HT-29 cells.

The involvement of YB-1 in EMT demonstrated in this study has been supported by previous studies using other cancer models and tumor types. In prostate cancer, research found that knockdown of YB-1 caused a 50\% reduction in cell invasion, and further investigation suggested that YB-1 regulated EMT by mammalian target of rapamycin and mitogen-activated protein kinase signaling $(9,27)$. In melanoma, the stimulating function of YB-1 in proliferation, invasion, apoptosis resistance and chemoresistance and the regulation of YB-1 in EMT-related signaling pathways have been widely proved $(29,30)$. Di Constanzo et al (31) demonstrated that YB-1 could enhance cell mobility and confer mesenchymal characteristics in squamous cancer cells. Furthermore, YB-1 is a transcriptional activator of the CD44 gene, which has been identified to enhance the invasion of CRC by EMT, thus adding further support towards a link between YB-1 and EMT in CRC $(32,33)$. Jürchott et al (27) suggested that CRC metastasis may be associated with the promotion of proliferation by YB-1. However, based on the results of the present study, it is speculated that this may additionally be due to EMT induction by YB-1.

Of note, there are different hypotheses regarding the function of YB-1. Although an clear pro-growth function of YB-1 has been widely proved in numerous types of tumor, such as lung cancer and osteosarcoma $(2,34)$, other studies have suggested that YB-1 may enhance EMT by inhibiting proliferation in breast cancer $(15,35,36)$. This difference is likely caused by the tissue specificity exhibited by the oncogenic function of YB-1; even within the same tumor, the role of YB-1 may be cell type-dependent (18). Other studies have found little association between YB-1 and EMT phenotype in gastric cancer, despite the role of YB-1 in promoting the invasion and migration of gastric cancer $(7,37)$. This may be due to differences in the experimental methods and evaluation criterions. Furthermore, to the best of our knowledge, there is no systematic and accurate evaluation guideline for defining EMT phenotype; this is also responsible for divergent conclusions.

In conclusion, the present study demonstrates that the expression of YB-1 is correlated with malignant tumor progression and an EMT phenotype in human CRC tissues. In vitro assays suggest that YB-1 may promote the proliferation, apoptosis resistance, invasion and migration of HT-29 cells by inducing EMT. These data enhance the current understanding of YB-1 in CRC and suggest that YB-1 may be a promising target for developing novel therapies for CRC. Further studies are now required to focus on the specific signaling network by which YB-1 regulates the EMT in CRC.

\section{Acknowledgements}

The authors would like to thank Professor Yu-Ping Gao (Department of Pathology, Renji Hospital, School of Medicine,
Shanghai Jiao Tong University) for her kind assistance in the immunohistochemistry assay. This study was financially supported by funding from the Science and Technology Commission of Shanghai Municipality (11ZR1427500).

\section{References}

1. Garcia-Albeniz X and Chan AT: Aspirin for the prevention of colorectal cancer. Best Pract Res Clin Gastroenterol 25: 461-472, 2011.

2. Lasham A, Samuel W, Cao H, et al: YB-1, the E2F pathway, and regulation of tumor cell growth. J Natl Cancer Inst 104: 133-146, 2012.

3. Bommert KS, Effenberger M, Leich E, et al: The feed-forward loop between YB-1 and MYC is essential for multiple myeloma cell survival. Leukemia 27: 441-450, 2013.

4. Takahashi M, Shimajiri S, Izumi H, et al: Y-box binding protein-1 is a novel molecular target for tumor vessels. Cancer Sci 101: $1367-1373,2010$

5. Lovett DH, Cheng S, Cape L, Pollock AS and Mertens PR: YB-1 alters MT1-MMP trafficking and stimulates MCF-7 breast tumor invasion and metastasis. Biochem Biophys Res Commun 398: 482-488, 2010.

6. Choi YK, Cho SG, Choi HS, Woo SM, Yun YJ, Shin YC and Ko SG: JNK1/2 activation by an extract from the roots of Morus alba L. reduces the viability of multidrug-resistant MCF-7/Dox cells by inhibiting YB-1-dependent MDR1 expression. Evid Based Complement Alternat Med 2013: 741985, 2013.

7. Wu Y, Yamada S, Izumi H, et al: Strong YB-1 expression is associated with liver metastasis progression and predicts shorter disease-free survival in advanced gastric cancer. J Surg Oncol 105: 724-730, 2012.

8. Panupinthu N, Yu S, Zhang D, et al: Self-reinforcing loop of amphiregulin and Y-box binding protein-1 contributes to poor outcomes in ovarian cancer. Oncogene: Jul 15, 2013 (Epub ahead of print).

9. Imada K, Shiota M, Kohashi K, et al: Mutual regulation between Raf/MEK/ERK signaling and Y-box-binding protein-1 promotes prostate cancer progression. Clin Cancer Res 19: 4638-4650, 2013.

10. Khan MI, Adhami VM, Lall RK, et al: YB-1 expression promotes epithelial-to-mesenchymal transition in prostate cancer that is inhibited by a small molecule fisetin. Oncotarget 5: 2462-2474, 2014.

11. Zhang H, Cheng S, Zhang M, et al: Prostaglandin E2 promotes hepatocellular carcinoma cell invasion through upregulation of YB-1 protein expression. Int J Oncol 44: 769-780, 2014.

12. Franco-Chuaire ML, Magda Carolina SC and Chuaire-Noack L: Epithelial-mesenchymal transition (EMT): principles and clinical impact in cancer therapy. Invest Clin 54: 186-205, 2013.

13. David JM and Rajasekaran AK: Dishonorable discharge: the oncogenic roles of cleaved E-cadherin fragments. Cancer Res 72: 2917-2923, 2012.

14. He X, Chen Z, Jia M and Zhao X: Downregulated E-cadherin expression indicates worse prognosis in Asian patients with colorectal cancer: evidence from meta-analysis. PLoS One 8: e70858, 2013.

15. Toiyama Y, Yasuda H, Saigusa S, Tanaka K, Inoue Y, Goel A and Kusunoki M: Increased expression of Slug and Vimentin as novel predictive biomarkers for lymph node metastasis and poor prognosis in colorectal cancer. Carcinogenesis: 34 : 2548-2557, 2013.

16. Todosi AM, Gavrilescu MM, Aniţei GM, Filip B and Scripcariu V: Colon cancer at molecular level - usefulness of epithelial-mesenchymal transition analysis. Rev Med Chir Soc Med Nat Iasi 116: 1106-1111, 2012.

17. Evdokimova V, Tognon C, Ng T, et al: Translational activation of snaill and other developmentally regulated transcription factors by YB-1 promotes an epithelial-mesenchymal transition. Cancer Cell 15: 402-415, 2009.

18. Shiota M, Yokomizo A, Itsumi M, et al: Twist1 and Y-box-binding protein-1 promote malignant potential in bladder cancer cells. BJU Int 108: E142-E149, 2011.

19. Matsushita K, Toiyama Y, Tanaka K, et al: Soluble CXCL16 in preoperative serum is a novel prognostic marker and predicts recurrence of liver metastases in colorectal cancer patients. Ann Surg Oncol 19 (Suppl 3): S518-S527, 2012. 
20. Tang FY, Pai MH and Chiang EP: Consumption of high-fat diet induces tumor progression and epithelial-mesenchymal transition of colorectal cancer in a mouse xenograft model. J Nutr Biochem 23: 1302-1313, 2012.

21. Wu HM, Cao W, Ye D, Ren GX, Wu YN and Guo W: Contactin 1 (CNTN1) expression associates with regional lymph node metastasis and is a novel predictor of prognosis in patients with oral squamous cell carcinoma. Mol Med Rep 6: 265-270, 2012.

22. Tiwari N, Gheldof A, Tatari M and Christofori G: EMT as the ultimate survival mechanism of cancer cells. Semin Cancer Biol 22: 194-207, 2012

23. Bhangu A, Wood G, Mirnezami A, Darzi A, Tekkis P and Goldin R: Epithelial mesenchymal transition in colorectal cancer: Seminal role in promoting disease progression and resistance to neoadjuvant therapy. Surg Oncol 21: 316-323, 2012.

24. Zhu QC, Gao RY, Wu W and Qin HL: Epithelial-mesenchymal transition and its role in the pathogenesis of colorectal cancer. Asian Pac J Cancer Prev 14: 2689-2698, 2013.

25. Lasham A, Print CG, Woolley AG, Dunn SE and Braithwaite AW: YB-1: oncoprotein, prognostic marker and therapeutic target? Biochem J 449: 11-23, 2013.

26. Tsofack SP, Garand C, Sereduk C, et al: NONO and RALY proteins are required for YB-1 oxaliplatin induced resistance in colon adenocarcinoma cell lines. Mol Cancer 10: 145, 2011.

27. Jürchott K, Kuban RJ, Krech T, et al: Identification of Y-box binding protein 1 as a core regulator of MEK/ERK pathway-dependent gene signatures in colorectal cancer cells. PLoS Genet 6: e1001231, 2010.

28. Hsieh AC, Liu Y, Edlind MP, et al: The translational landscape of mTOR signalling steers cancer initiation and metastasis. Nature 485: 55-61, 2012.
29. Schittek B, Psenner K, Sauer B, et al: The increased expression of Y box-binding protein 1 in melanoma stimulates proliferation and tumor invasion, antagonizes apoptosis and enhances chemoresistance. Int J Cancer 120: 2110-2118, 2007.

30. Sinnberg T, Sauer B, Holm P, Spangler B, Kuphal S, Bosserfhoff A and Schittek B: MAPK and PI3K/AKT mediated YB-1 activation promotes melanoma cell proliferation which is counteracted by an autoregulatory loop. Exp Dermatol 21: 265-270, 2012.

31. Di Costanzo A, Troiano A, di Martino O, et al: The p63 protein isoform $\Delta \mathrm{Np} 63 \alpha$ modulates Y-box binding protein 1 in its subcellular distribution and regulation of cell survival and motility genes. J Biol Chem 287: 30170-30180, 2012.

32. To K, Fotovati A, Reipas KM, et al: Y-box binding protein-1 induces the expression of CD44 and CD49f leading to enhanced self-renewal, mammosphere growth, and drug resistance. Cancer Res 70: 2840-2851, 2010.

33. Cho SH, Park YS, Kim HJ, et al: CD44 enhances the epithelial-mesenchymal transition in association with colon cancer invasion. Int J Oncol 41: 211-218, 2012.

34. Fujiwara-Okada Y, Matsumoto Y, Fukushi J, et al: Y-box binding protein-1 regulates cell proliferation and is associated with clinical outcomes of osteosarcoma. Br J Cancer 108: 836-847, 2013.

35. Evdokimova V, Tognon C, Ng T and Sorensen PH: Reduced proliferation and enhanced migration: two sides of the same coin? Molecular mechanisms of metastatic progression by YB-1. Cell Cycle 8: 2901-2906, 2009.

36. Mouneimne G and Brugge JS: YB-1 translational control of epithelial-mesenchyme transition. Cancer Cell 15: 357-359, 2009.

37. Guo TT, Yu YN, Yip GW, Matsumoto K and Bay BH: Silencing the YB-1 gene inhibits cell migration in gastric cancer in vitro. Anat Rec (Hoboken) 296: 891-898, 2013. 